\title{
De Médico de Família a Preceptor de Estudantes no Sistema Único de Saúde: Relato de Experiência
}

\section{From Family Doctor to Student Preceptor in the Brazilian Unified Health System: Experience Report}

Daniel Almeida de Oliveira ${ }^{I}(\mathbb{D}$ Eder Viana de Souza $a^{I}$ (D)

\section{PALAVRAS-CHAVE}

- Atenção Primária à Saúde.

- Aprendizagem.

- Medicina.

- Saúde da Família
Introdução: Neste artigo, é relatada a experiência de um médico especialista em Medicina de Família e Comunidade, que trabalha em uma unidade básica de saúde da região sul do município de São Paulo, na atenção primária à saúde do Sistema Único de Saúde, ao se tornar preceptor de estudantes de Medicina da Universidade São Caetano do Sul. Método: Os estudantes conheceram a estrutura típica de uma unidade básica de saúde e treinaram a confecção da entrevista médica, sob supervisão, e do exame físico, à medida que adquiriam as competências necessárias. Treinaram ainda o pedido de exames complementares. Com isso aprenderam na prática a aplicar o método clínico. Os estudantes também treinaram a competência de aplicar o método clínico em ambientes não controlados por meio da visita domiciliar, o que lhes permitiu também conhecer a realidade do paciente in loco. Por meio do acompanhamento de famílias e de casos-índice realizado durante alguns anos, os estudantes puderam conhecer os diferentes ciclos de atendimento da Estratégia Saúde da Família, inclusive a questão da morte, que se inicia no contexto sociofamiliar. Os discentes também atuaram em grupos de orientação e transmissão de conhecimento para a comunidade e puderam treinar a comunicação e a adaptação referentes à população atendida. Participaram de reuniões de equipe, nas quais vivenciaram o planejamento semanal das atividades, a discussão interdisciplinar de casos mais complexos e as estratégias criadas. Resultados: Obtiveram-se resultados promissores com o uso das metodologias ativas que se baseiam na autonomia do estudante ante o processo de aprendizagem. Essas metodologias são aplicadas ao ensino da Medicina em ambientes de prática clínica, pois o contato com a realidade melhora o aprendizado, desafia o aluno a pesquisar com autonomia a fim de entender com clareza o que fazer com os objetivos de aprendizagem estabelecidos, ensina a usar experiências pregressas para inter-relacionar novos conhecimentos com os conhecimentos prévios, por meio da Medicina Baseada em Evidências, e refletir sobre a prática médica. Conclusão: De acordo com as Diretrizes Nacionais Curriculares, a inserção do estudante de Medicina no âmbito do Sistema Único de Saúde é imprescindível para que ele possa adquirir os conhecimentos necessários para atuar sobretudo na atenção primária à saúde. 


\section{KEYWORDS}

- Primary Health Care.

- Learning.

- Medicine.

- Family Health.

\section{ABSTRACT}

Introduction: This article reports the experience of a physician, specialist in Family and Community Medicine, who works in a Basic Health Unit in the southern region of the city of São Paulo, in the Primary Health Care of the Unified Health System, when he became a Preceptor of Medical students at Universidade São Caetano do Sul. Method: The students learned about the typical structure of a Basic Health Unit. They learned how to acquire the medical history during anamnesis, under supervision, and the physical examination, as they acquired the necessary skills. They also learned how to request complementary examinations. With this training, they learned how to apply the clinical method during practice. The students also learned the competence to apply the clinical method in uncontrolled environments through the home visit, which also made it possible to know the reality of the patient in loco. The follow-up of families and the index cases for a few years gave the students the opportunity to experience the different care cycles of the Family Health Strategy, including the question of death, which started in the socio-family context. They also participated and carried out assistance and knowledge transmission groups for the community, in which students were able to train communication and adaptation at the population level. They met and participated in the team meetings, which allowed showing the weekly planning of activities, the interdisciplinary discussion of more complex cases and the created strategies. Results: The promising results of the Active Methodologies based on student autonomy, in relation to the learning process, are applied to the teaching of Medicine in clinical practice environments, since the contact with reality improves learning, challenges the students to research and reflect with autonomy to think about what they must do with the established learning goals and teaches them to use previous experiences to interrelate new knowledge with previous information through Evidence-Based Medicine and reflect on medical practice. Conclusion: Comments are also made on the National Curricular Guidelines, which request the inclusion of the medical student within the scope of the Unified Health System, aimed mainly at Primary Health Care learning.

Recebido em 9/9/19

Aceito em 23/7/20

\section{INTRODUÇÃO}

As Diretrizes Curriculares Nacionais (DCN) deixam clara a inserção do estudante de Medicina no âmbito do Sistema Único de Saúde (SUS) para aprendizado principalmente na atenção primária à saúde (APS) e tornam flexíveis os currículos, o que permitirá que os discentes se adaptem ao conhecimento científico atual ${ }^{1-7}$.

Com o intuito de oferecer ao estudante maior autonomia ante o processo de aprendizagem, as metodologias ativas propõem os seguintes objetivos: levar o discente a "aprender a aprender",8,-12, desafiá-lo a realizar de forma autônoma pesquisas para que possa entender com clareza o que fazer com os objetivos de aprendizagem estabelecidos, ensiná-lo a usar experiências pregressas para inter-relacionar novos conhecimentos com os conhecimentos prévios, despertar a curiosidade e incentivá-lo a sugerir procedimentos novos que não foram considerados nas aulas e pelo professor ${ }^{1,5,8,9,12}$. O aprendizado deveria estar centrado no estudante e na pessoa, o que levaria o discente a um maior interesse por aprender sobre a pessoa, e não somente sobre a doença ${ }^{1,3,5,8,9,11,12}$. Incrementaria a relação do aluno com os outros estudantes, os professores e as pessoas envolvidas. O currículo deveria ser integrado sem a divisória entre ciclo básico e profissionalizante (o estudante idealmente tem contato com a prática desde o primeiro ano, preferencialmente no SUS, em que aprende não só o método clínico, mas também o funcionamento do sistema ${ }^{1-9,12}$. Isso aumentaria a resolubilidade clínica ao fazer o estudante lidar com condições e problemas complexos e singulares de saúde de forma contínua e longitudinal ${ }^{5-7}$, por meio da Medicina Baseada em Evidências ${ }^{9-12}$. Didaticamente, foi comprovado que o contato com a realidade melhora o aprendizado, a despeito do estudo da mera teoria ${ }^{3}$.

\section{MATERIAIS E MÉTODOS}

Trata-se de relato de experiência pessoal de transição de um médico de família e comunidade a preceptor de estudantes de Medicina inseridos no contexto da APS no SUS.

\section{RESULTADOS}

Em 2016, fui convidado pela Associação Saúde da Família para ser preceptor dos estudantes da Universidade São Caetano do Sul.

No início, foi um trabalho árduo, pois desconhecia o método ativo da universidade. Aos poucos, por meio de um curso e de estudos autônomos, pude conhecer a metodologia ativa.

Os estagiários são estudantes de Medicina de uma universidade pública municipal que pagam mensalidade. A maioria dos estudantes é de classe mais abastada e nunca utilizou o SUS. Tal realidade é uma grande chance de mostrar realmente a função do SUS e retirar de vez estigmas, como o fato de o SUS ser um serviço para pobres.

Primeiramente, os estudantes conheceram a estrutura típica de uma unidade básica de saúde e permaneceram algum tempo na recepção, sala de vacinas, sala de curativos, sala de acolhimento, sala de saúde da mulher e colheita de exame preventivo do câncer de colo uterino, farmácia etc.

A unidade básica de saúde, na Estratégia Saúde da Família, funciona como porta de entrada do SUS e deveria funcionar também como a gestora das redes de atenção à saúde ${ }^{13}$.

Aos poucos, os estudantes começaram a fazer a entrevista médica sob supervisão. Eu os direcionava e completava com perguntas pertinentes quando saíam do objetivo principal e faltava-lhes o conhecimento.

Quanto ao exame físico, à medida que adquiriam as competências

REVISTA BRASILEIRA DE EDUCAÇÃO MÉDICA

2 44 (4) : e116; 2020 
necessárias, foi sendo feito por eles e revisado por mim.

Em relação aos exames complementares, muitas vezes os estudantes sugeriam alguns, e eu acrescentava e explicava o porquê de cada exame.

Com isso, o método clínico de formulação de hipóteses diagnósticas, análise da probabilidade de cada hipótese, solicitação de exames complementares e retorno do paciente, com nova entrevista médica e exame físico, muitas vezes após tratamento em relação ao diagnóstico de maior probabilidade baseado em evidências, era testado. As probabilidades eram ajustadas segundo a eficácia do tratamento e os exames complementares.

A reflexão da prática médica se mostra um importante fator para melhorar e aprofundar o aprendizado dos estudantes. A reflexão envolve pensamento crítico, exploração das experiências emocionais e pessoais, e exame do impacto das ações ${ }^{14}$. O pensamento crítico é despertado no ensino do método clínico baseado em evidências, na confecção de um projeto terapêutico singular e na adaptação e avaliação desse projeto na evolução do caso. A exploração das experiências emocionais e pessoais é obtido por meio de discussão com o estudante, para que ele possa verbalizar tais experiências e para que tome consciência disso. $\mathrm{O}$ exame do impacto das ações é feito tanto no aprendizado e conhecimento do aluno quanto na melhora da saúde do indivíduo acompanhado.

A visita domiciliar, procedimento singular da Estratégia Saúde da Família, é um momento em que podemos testar o método clínico na realidade do paciente. A visita domiciliar, além de gerar um "choque de realidade" nos estudantes (muitos nunca haviam ido a uma comunidade - "favela" - e visto condições tão precárias de habitação), proporcionou a competência de aplicar o método clínico em ambientes não controlados.

Cada aluno acompanhava uma família durante o estágio. As famílias eram diversificadas, e havia pessoas nos diferentes ciclos de vida: gestantes, crianças pequenas, jovens e adultos, idosos, de ambos os sexos. O acompanhamento de famílias e de casos-índice realizado durante alguns anos permitiu que os estudantes conhecessem os diferentes ciclos de atendimento da Estratégia Saúde da Família. Por exemplo, mostroulhes mulheres em idade fértil que engravidaram, passaram pelo puerpério e pela puericultura; pacientes com fatores de risco como diabetes e hipertensão arterial que evoluíram para insuficiência renal, acabando em hemodiálise; infartados que tiveram de ser aposentados e um caso de óbito por infarto. A morte, no método tradicional da medicina, tem sua iniciação no contato com o cadáver para a dissecação. $\mathrm{Na}$ metodologia ativa, inicia-se no contexto sociofamiliar, mostrando não somente um "cadáver frio", mas também uma pessoa de quem os parentes e amigos sentem falta e que demonstram seus sentimentos. É um aspecto mais humano de ver a morte, evento inevitável na medicina.

Os grupos de orientação e transmissão de conhecimento para a comunidade foram um espaço rico para a ação dos estudantes, que puderam treinar a comunicação, tornando-a acessível à população, sanando dúvidas e resolvendo pequenos problemas, sob supervisão.

O papel do preceptor em relação à perspectiva do docente está associado à prática médica em situações reais, sob supervisão, mas com autonomia. Ao lidarem com a realidade da prática médica em espaços não controlados como uma unidade básica de saúde de Estratégia Saúde da Família, o estudante exercita a teoria aprendida na prática e adquire as competências necessárias para ser um médico generalista.

A participação dos estudantes na reunião de equipe possibilitou mostrar o planejamento semanal das atividades, a discussão interprofissional de casos mais complexos, as estratégias criadas por meio do diálogo dos muitos saberes dos diversos profissionais da saúde que participaram, como o enfermeiro e os agentes comunitários de saúde.

\section{CONCLUSÃO}

O estágio na APS proporcionou aos estudantes autonomia e conhecimento ligado à realidade. Para mim, que sou preceptor, foi uma experiência frutífera, gratificante e de muita aprendizagem, pois, quando se ensina, aprende-se mais.

\section{AGRADECIMENTO}

À Associação Saúde da Família, por apoiar nas UBS a Preceptoria.

\section{REFERÊNCIAS}

1. Brasil. Ministério da Educação. Conselho Nacional de Educação. Câmara de Educação Superior. Resolução nº 3, de 20 de junho de 2014. Institui Diretrizes Curriculares Nacionais do Curso de Graduação em Medicina e dá outras providências [acesso em 29 dez 2018]. Disponível em: http://portal.mec.gov.br/index.php?option=com docman \&view =download\&alias $=15874-$ rces003 $-14 \&$ category slug=junho-2014-pdf\&Itemid=30192.

2. Anjos RMP, Gianini RJ, Minari FC, de Luca AHS, Rodrigues MP. "Vivendo o SUS": uma experiência prática no cenário da atenção básica. Rev Bras Educ Med. 2010;34(1):172-83.

3. Adler MS, Gallian DMC. Formação médica e serviço único de saúde: propostas e práticas descritas na literatura especializada. Rev Bras Educ Med. 2014;38(3):388-96.

4. Ciuffo RS, Ribeiro VMB. Sistema Único de Saúde e a formação dos médicos: um diálogo possível? Interface Comun. Saúde Educ. 2008;12(24):125-40.

5. Demarzo MMP, Almeida RCC, Marins JJN, Trindade TG, Anderson MIP Stein AT, et al. Diretrizes para o ensino na atenção primária à saúde na graduação em medicina. Rev Bras Educ Med. 2012;36(1):143-8.

6. Ferreira RC, Fiorini VML, Crivelaro E. Formação profissional no SUS: o papel da atenção básica em saúde na perspectiva docente. Rev Bras Educ Med. 2010;34(2):207-15.

7. Ferreira RC, Silva RF, Aguera CB. Formação do profissional médico: a aprendizagem na atenção básica de saúde. Rev Bras Educ Med. 2007;31(1):52-9.

8. Costa JRB, Romano VF, Costa RR, Gomes AP, Batista RS. Active teaching-learning methodologies: medical students' views of problembased learning. Rev Bras Educ Med. 2011;35(1):13-9.

9. Spanhol FJ, Farias GF, Souza MV, organizadores. EAD, PBL e o desafio da educação em rede: metodologias ativas e outras práticas na formação do educador coinvestigador. São Paulo: Blucher; 2018. 242 p.

10. El Dib RP. How to practice evidence-based medicine. J Vasc Bras. 2007;6(1):1-4.

11. Dent JA. AMEE Guide $n^{\circ}$ 26: clinical teaching in ambulatory care settings: making the most of learning opportunities with outpatients Med Teach. 2005;27(4):302-15.

12. Kozanitis A. Activités pour encourager l'apprentissage actif durant les cours. Bureau d’Appui Pédagogique. Polytechnique Montréal; 2005.

13. Mendes EV. As redes de atenção à saúde. Ciênc Saúde Colet. 2010;15(5):2297-305.

REVISTA BRASILEIRA DE EDUCAÇÃO MÉDICA

3 44 (4) : e116; 2020 
14. Winkel AF, Yingling S, Jones AA, Nicholson J. Reflection as a learning tool in graduate medical education: a systematic review. Journal of Graduate Medical Education. 2017;9(4):430-9.

\section{CONTRIBUIÇÃO DOS AUTORES}

Daniel Almeida de Oliveira foi o autor principal e Eder Viana de Souza foi o coautor e orientador.

\section{CONFLITO DE INTERESSES}

Os autores declaram não haver conflito de interesses neste estudo.

\section{ENDEREÇO PARA CORRESPONDÊNCIA}

Daniel Almeida de Oliveira. Rua Treze de Maio, 681, Bela Vista, São Paulo, SP, Brasil. CEP: 01327-000.

E-mail: 4477dao@gmail.com 